VILLAS BÔAS, G.L.; CASTELO BRANCO, M.; MEDEIROS, M.A.; MONNERAT, R.G.; FRANÇA, F.H. Inseticidas para o controle da traça-das-crucíferas e impactos sobre a população natural de parasitóides. Horticultura Brasileira, Brasília, v.22, n.4, p.696-699, out-dez 2004.

\title{
Inseticidas para o controle da traça-das-crucíferas e impactos sobre a população natural de parasitóides
}

\author{
Geni L. Villas Bôas ${ }^{1}$; Marina Castelo Branco ${ }^{1}$; Maria Alice de Medeiros ${ }^{1}$; Rose G. Monnerat ${ }^{2}$; Félix \\ Humberto França ${ }^{1}$

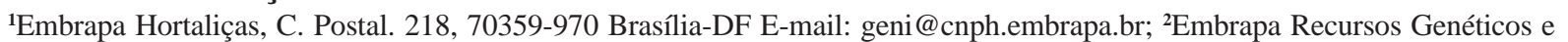 \\ Biotecnologia
}

\section{RESUMO}

A traça-das-crucíferas (TDC) Plutella xylostella (Lepidoptera: Yponomeutidae) é a principal praga da cultura do repolho e seu controle é feito através de inseticidas, com possíveis efeitos adversos na população natural dos seus parasitóides. Nos últimos anos, novos ingredientes ativos foram colocados no mercado, tornando necessária a avaliação da eficiência destes produtos e o impacto na população de parasitóides. Com este objetivo, foi instalado um experimento, de junho a outubro de 2001, na área experimental da Embrapa Hortaliças, com a cultivar Kenzan. Foram utilizados os produtos Spinosad (38,4 g i.a./ha), Thiacloprid (96 g i.a./ha), Indoxacarb (24 g i.a./ha), Bacillus thuringiensis var. kurstaki (16,8 g i.a./ha), uma nova cepa (S764, concentração de esporos 4 x 10 E 9) de $B$. thuringiensis (168 g i.a./ha), disponibilizada para teste pela Embrapa Recursos Genéticos e Biotecnologia e uma testemunha, sem pulverização. As pulverizações, num total de oito, foram semanais, iniciando-se a partir da formação das cabeças de repolho, quatro semanas após o transplante. Foram efetuadas amostragens semanais em cinco plantas por parcela, contando-se o número de furos e larvas nas quatro folhas centrais da cabeça do repolho. Avaliou-se também o número de larvas na parte externa da cabeça do repolho. A cada 15 dias foram efetuadas coletas de larvas (folhas centrais e externas), que eram levadas ao laboratório para avaliação de parasitismo. Por ocasião da colheita, as cabeças foram avaliadas quanto ao valor comercial, através de notas e porcentagem de cabeças comerciais, além da contagem do número de furos, larvas na cabeça e larvas na planta toda. Os produtos Spinosad, Indoxacarb e B. thuringiensis var. kurstaki foram os mais eficientes para o controle de P. xylostella e não diferiram entre si para os parâmetros avaliados. A utilização desses produtos propiciou a produção de cabeças de repolho com alto padrão comercial. Os produtos Thiacloprid e a cepa de $B$. thuringiensis S764 (Embrapa Recursos Genéticos e Biotecnologia) não foram eficientes. O B. thuringiensis var. kurstaki conciliou eficiência de controle com baixo impacto na população de parasitóides. O produto Spinosad apresentou impacto na população de parasitóides, quando foram amostradas larvas de $P$. xylostella da cabeça do repolho. As espécies de parasitóides coletadas com maior frequiência foram Diadegma leontiniae; Apanteles sp.; Actia sp. e Oomyzus sokolowskii.

Palavras-chave: Plutella xylostella, repolho, inimigo natural, controle químico.

\begin{abstract}
Insecticide evaluation for control of the diamondback moth and impact on natural populations of parasitoids associated with
\end{abstract} diamondback moth

The Diamondback moth (DBM) Plutella xylostella (Lepidoptera: Yponomeutidae) is the most important insect pest of cabbage in Brazil, which is controlled through pesticides sprays, that have adverse effects on the parasitoid natural population. In the last few years, new active ingredients were launched in the market, bringing the need to evaluate their agronomical efficiency and impact on natural enemies. An experiment was set up from June to October 2001, at Embrapa Hortaliças (Brazil), using the cabbage cultivar "Kenzan". The treatments were sprayed weekly, during eight weeks, starting when cabbage heads were being formed (about four weeks after transplanting). Five plants in each plot were sampled each week and the number of holes and larvae in the four central leaves in each cabbage head were recorded. The number of holes and larvae also were recorded for the whole cabbage plant. Every 15 days, larvae were collected from the plant and taken to the laboratory for parasitism evaluation. At the harvest, cabbage heads were evaluated regarding their commercial value, established based on a scale of grades and percent of commercial heads, besides counting the number of holes and larvae located at the cabbage head and in the whole plant. Among the pesticides tested, Spinosad, Indoxacarb and $B$. thuringiensis var. kurstaki were the most efficient in DBM control and did not differ from each other in all the parameters evaluated, and these products provided cabbage heads with great commercial value. The pesticides Thiacloprid and a strain of B.T. S764 (Embrapa) were not efficient. However, B. thuringiensis var. kurstaki reunited efficiency of control and low impact in the population of parasitoid. The pesticide Spinosad caused impact in the parasitoid population in the larvae of DBM located in the cabbage head. The most frequent parasitoid collected in the experimental area were Diadegma leontiniae; Apanteles sp.; Actia sp. and Oomyzus sokolowskii.

Keywords: Plutella xylostella, cabbage, natural enemies, chemical control.

(Recebido para publicação em 25 de novembro de 2003 e aceito em 13 de setembro de 2004)

\begin{abstract}
A traça-das-crucíferas (TDC) Plutella xylostella (Lepidoptera: Yponomeutidae) é a principal praga da cultura do repolho, sendo que o controle químico é o método mais empregado
\end{abstract}

para reduzir os danos desta praga. Em geral, utiliza-se grande número de aplicações por ciclo da cultura, podendo chegar a 15/20, independente da presença da praga no campo (Guan-Soon,
1990; Carballo, 1992; Sampson, 1992). No Brasil, observou-se que o número de aplicações pode variar de um a quatro por semana (Castelo Branco et al., 2001). Diferentes ingredientes ativos, 
isolados ou em mistura, são utilizados nas lavouras, com possíveis efeitos adversos sobre a população natural de parasitóides (Castelo Branco e Medeiros, 2001), além de acelerar a seleção de populações resistentes aos produtos empregados (Castelo Branco e Gatehouse, 1997, 2001).

Para reduzir o uso indiscriminado do controle químico, foi proposto pela Embrapa Hortaliças um programa de manejo integrado da traça-dascrucíferas (Castelo Branco et al., 1996), que vem sendo adotado por diversos produtores da região, com resultados positivos (Oliveira et al., 2000). Este manejo envolve a utilização da tecnologia denominada nível de dano econômico (Castelo Branco et al., 1996); cultivares tolerantes; plantios e colheitas coordenados; manejo de resistência a inseticidas (Castelo Branco e Gatehouse, 1997; Castelo Branco et al., 2003) e controle químico, sendo que o principal produto empregado é o Bacillus thuringiensis (BT), por ser um produto seletivo para inimigos naturais (Monnerat, 1995; França e Medeiros, 1998).

O manejo integrado é um processo que prevê uma contínua incorporação de novas tecnologias, que permitam uma redução significativa do uso de inseticidas em lavouras de brássicas. Deste modo, a identificação da eficiência dos novos inseticidas e a avaliação da seletividade a inimigos naturais é importante, sendo o objetivo deste trabalho.

\section{MATERIAL E MÉTODOS}

O experimento foi conduzido de junho a outubro de 2001, em Brasília, época de maior densidade populacional da TDC, na área experimental da Embrapa Hortaliças. A cultivar empregada foi Kenzan e os tratamentos foram: Spinosad (38,4 g i.a./ha), Thiacloprid (96 g i.a./ha), Indoxacarb (24 g i.a./ha), B. thuringiensis var. kurstaki (16,8 g i.a./ ha), uma nova cepa (S764, concentração de esporos 4 x 10 E 9) de $B$. thuringiensis (168 g i.a./ha), disponibilizada para teste pela Embrapa Recursos Genéticos e Biotecnologia e uma testemunha, sem pulverização. O delineamento experimental foi de blocos ao acaso, com quatro repetições.

Tabela 1. Número de furos, larvas de TDC nas quatro folhas centrais (FC) e na planta toda (PT), amostrados na colheita; nota e porcentagem de cabeças comerciais de repolho. Brasília, Embrapa Hortaliças, 2001.

\begin{tabular}{|c|c|c|c|c|c|}
\hline $\begin{array}{c}\text { Tratamentos } \\
\text { (g i.a./ha) }\end{array}$ & $N^{\circ}$ furos & $\begin{array}{c}\mathrm{N}^{\circ} \text { larvas } \\
\text { (FC) }\end{array}$ & $\begin{array}{l}\mathrm{N}^{\circ} \text { larvas } \\
\text { (PT) }\end{array}$ & Nota & $\begin{array}{l}\% \text { Cabeças } \\
\text { comerciais }\end{array}$ \\
\hline Spinosad $(38,4)$ & $2,1 a^{1}$ & $0,0 \mathrm{a}$ & $0,3 a$ & $1,3 \mathrm{a}$ & 100,0 a \\
\hline Indoxacarb $(24,0)$ & 3,7 a & 0,1 a & $0,3 a$ & 1,4 a & 95,8 a \\
\hline $\begin{array}{l}\text { Bacillus thuringiensis } \\
\text { var. kurstaki }(16,8)\end{array}$ & 3,9 a & 0,1 a & 0,3 a & 1,4 a & 95,8 a \\
\hline Thiacloprid $(96,0)$ & 31,3 & 0,5 & $0,5 \mathrm{a}$ & 2,7 & $41,7 \quad b$ \\
\hline $\begin{array}{l}\text { Cepa B. thuringiensis } \\
(168,0)\end{array}$ & 38,9 & 0,4 & 0,5 a & 2,9 & $37,5 \quad b$ \\
\hline Testemunha & 53,3 & 0,8 & $1,3 \quad b$ & 3,2 & 16,7 \\
\hline
\end{tabular}

${ }^{1}$ Dados originais, para análise estatística foram transformados em $\sqrt{x+0,5}$. Médias seguidas da mesma letra, na coluna, não diferem entre si, pelo teste DMS a 5\%.

Tabela 2. Impacto de inseticidas sobre parasitóides de TDC, medido em larvas coletadas nas quatro folhas centrais e folhas externas da cabeça do repolho. Brasília, Embrapa Hortaliças, 2001.

\begin{tabular}{|c|c|c|c|c|c|}
\hline $\begin{array}{l}\text { Tratamentos } \\
\text { (g i.a./ha) }\end{array}$ & $\begin{array}{c}\text { Folhas centrais } \\
\text { Média } \pm E^{2} M^{1}\end{array}$ & $\begin{array}{l}\text { Folhas } \\
\text { externas } \\
\text { Média } \pm \\
\text { EPM }\end{array}$ & $\begin{array}{l}\text { Diadegma } \\
\text { leontiniae }\end{array}$ & $\begin{array}{l}\text { Apanteles } \\
\text { sp. }\end{array}$ & $\begin{array}{c}\text { Outros } \\
\text { parasitóides }\end{array}$ \\
\hline Spinosad $(38,4)$ & $16,2 \pm 9,9 \quad c^{2}$ & $44,2 \pm 13,0$ & 11,2 & 27,0 & 1,1 \\
\hline Indoxacarb $(24,0)$ & $22,8 \pm 9,6 \quad b c$ & $62,2 \pm 9,6$ & 6,6 & 33,1 & 2,5 \\
\hline $\begin{array}{l}\text { Bacillus thuringiensis } \\
\text { var. kurstaki }(16,8)\end{array}$ & $51,0 \pm 10,0 a$ & $64,4 \pm 10,3$ & 3,7 & 32,4 & 4,4 \\
\hline Thiacloprid $(96,0)$ & $58,8 \pm 8,4 a$ & $55,8 \pm 6,6$ & 9,0 & 31,9 & 11,4 \\
\hline $\begin{array}{l}\text { Cepa } B \text {. } \\
\text { thuringiensis }(168,0)\end{array}$ & $38,8 \pm 10,2 a b c$ & $65,8 \pm 9,4$ & 6,2 & 24,9 & 8,0 \\
\hline Testemunha & $47,6 \pm 9,0 a b$ & $49,2 \pm 9,4$ & 6,8 & 19,8 & 9,7 \\
\hline
\end{tabular}

${ }^{1}$ Média e erro padrão da média; ${ }^{2}$ Dados originais, para análise estatística foram transformados em $\sqrt{x+0,5}$. Médias seguidas da mesma letra, na coluna, não diferem entre si, pelo teste DMS a $5 \%$.

Cada parcela foi constituída de duas linhas de repolho com 10 plantas cada uma. As pulverizações, num total de oito, foram semanais, iniciando-se a partir da formação das cabeças de repolho, quatro semanas após o transplante.

Foram efetuadas amostragens semanais em cinco plantas por parcela, contando-se o número de furos e larvas nas quatro folhas centrais da cabeça do repolho. Avaliou-se também o número de larvas na parte externa da cabeça. A cada 15 dias foram efetuadas coletas de larvas (folhas centrais e externas), que eram levadas para o laboratório e criadas até a fase de pupa, em câmaras BOD $\left(25 \pm 2^{\circ} \mathrm{C}, 70 \%\right.$ UR e $\left.14 \mathrm{~h} \mathrm{luz}\right)$ para avaliação do índice de parasitismo. Por ocasião da colheita, as cabeças foram avaliadas quanto ao valor comercial, através de notas ( 1 = folhas raspadas ou sem dano; 2 = folhas com furos pequenos; 3 = folhas com furos grandes; 4 = plantas com folhas totalmente danificadas) e porcentagem de cabeças comerciais (que receberam notas 1 e 2), além da contagem do número de furos, larvas na cabeça e larvas na planta toda (Castelo Branco et al., 1996).

\section{RESULTADOS E DISCUSSÃO}

Spinosad, Indoxacarb e $B$. thuringiensis var. kurstaki foram os produtos mais eficientes para o controle de P. xylostella e não diferiram entre si em número de furos e larvas, nota e porcentagem de cabeças comerciais (Tabela 1). Os parâmetros nota e número de furos apresentaram-se adequados para a identificação dos melhores inseticidas (Tabela 1), corroborando os resultados 
de Villas Bôas et al. (1990). No entanto, a contagem de larvas na planta toda não foi um parâmetro adequado neste experimento, por não se relacionar com o aproveitamento comercial de cabeças de repolho (Tabela 1). Thiacloprid e a cepa de B. thuringiensis S764 (Embrapa Recursos Genéticos e Biotecnologia) não foram eficientes no controle de $P$. xylostella (Tabela 1), embora tenham apresentado resultados significativamente superiores à testemunha. Ainda que Thiacloprid não tenha sido eficiente para o controle de TDC, este inseticida foi eficiente para outros lepidópteros como Helicoverpa zea (Castelo Branco et al., 2003). A cepa (S764) de B. thuringiensis testada, por não ter sofrido nenhum processo de formulação, mostrou-se inadequada para ser utilizada diretamente em campo, uma vez que a toxina é muito sensível a radiação solar. Em testes de laboratório esta cepa foi eficiente, sendo que a mortalidade de TDC foi próximo de $100 \%$ (Monnerat, 2003, comunicação pessoal).

O produto Spinosad apresentou o maior impacto na população de parasitóides, quando foram amostradas larvas de $P$. xylostella nas quatro folhas centrais da cabeça do repolho (Tabela 2). Ainda que a taxa de parasitismo das larvas coletadas nas folhas externas não tenha apresentado diferença significativa entre os tratamentos, ela foi elevada, variando de 44 a $66 \%$, enquanto nas folhas centrais variou de 16 a $60 \%$ (Tabela 2). Castelo Branco (1997) observou que os inseticidas, quando aplicados sobre as plantas de repolho atingem as folhas centrais, porém as partes inferiores das folhas externas não são atingidas. Como as larvas de TDC são encontradas nas cabeças de repolho ou na parte inferior das folhas externas (Castelo Branco e Medeiros, 2001), verifica-se que esta distribuição de larvas sobre as plantas permite a maior sobrevivência dos parasitóides nas áreas externas das plantas tratadas.

As espécies de parasitóides coletadas com maior frequiência foram Diadegma leontiniae; Apanteles sp.; Actia sp.; Oomyzus sokolowskii e algumas outras espécies (Tabela 2). Levantamentos realizados em 1995, $1996 \mathrm{e}$ 1998 na Embrapa Hortaliças identifica- ram o parasitóide Diadegma sp. como o mais abundante nos meses de abril a agosto e foi observado um parasitismo elevado na parte externa da planta (Castelo Branco e Medeiros, 2001). Por outro lado, Apanteles sp. predomina nos meses de outubro a novembro sob temperatura elevada, apresentando até $83 \%$ de parasitismo, sendo praticamente ausente no período mais frio do ano, de abril a agosto.

Tem sido observado, em áreas de uso intensivo de inseticidas, índices de parasitismo de até $86 \%$, devido a um escape das larvas e parasitóides aos inseticidas, uma vez que a maioria dos produtos não possui ação translaminar e não consegue atingir uma parte da população de larvas e parasitóides localizados na parte inferior da folha (Castelo Branco e França, 2001). Parasitóides como D. semiclausum, em condições de campo, exibem alta capacidade de locomoção e resistência parcial aos inseticidas (Takashino et al., 2001). De maneira semelhante, Idris e Grafius (1993) verificaram que o parasitismo por $D$. insulare não foi afetado pelos tratamentos, seis dias após a pulverização, sugerindo que o parasitismo pode ser influenciado pela vegetação presente na área. De maneira diversa, Hill e Foster (2000) observaram que Spinosad causou $100 \%$ de mortalidade a $D$. insulare, e BT foi considerado não tóxico. Em pragas de algodão, Indoxacarb e Spinosad foram seletivos para inimigos naturais, embora Spinosad tenha se mostrado tóxico para Trichogramma pretiosum e moderadamente tóxico para Cotesia marginiventris (Ruberson e Tillman, 1999). Spinosad apresentou boa seletividade para insetos polinizadores e ácaros predadores, e algum efeito tóxico em himenópteros parasitóides, embora de pouca duração, devido à sua baixa persistência (Miles e Dutton, 2000).

$\mathrm{O}$ produto $B$. thuringiensis var. kurstaki conciliou alta eficiência de controle com baixo impacto no nível de parasitismo, sendo que sua utilização deve ser enfatizada em programas de manejo integrado de pragas. Embora com algum impacto na população de parasitóides, os produtos Spinosad e Indoxacarb se mostraram eficientes no controle da TDC, e podem ser utiliza- dos como opções para a rotação de inseticidas, onde se preconiza a rotação de produtos de diferentes grupos químicos. De acordo com o comportamento das larvas de TDC e seus parasitóides, reforça-se aqui a recomendação de se direcionar as pulverizações para as folhas centrais da cabeça do repolho, permitindo assim um escape dos parasitóides nas folhas externas.

\section{AGRADECIMENTOS}

Aos ex-bolsistas da Embrapa Hortaliças (estudantes da Universidade Católica de Brasília) João A.O. de Paulo e Cibila S. Simplício, pela inestimável ajuda na coleta e tabulação dos dados. Ao Sr. Hozanan P. Chaves pelo auxílio nos trabalhos de campo.

\section{LITERATURA CITADA}

CARBALLO, V.M. Investigación sobre manejo integrado de Plutella xylostella L. en el cultivo de repolho en Costa Rica. Cerba, v.33, p.617-622, 1992.

CASTELO BRANCO, M. Insecticide resistance in Plutella xylostella (Linnaeus) (Yponomeutidae) in the Federal District, Brazil; effects of local and longrange movement of months. Bangor: University of Wales, 1997. 196 p. (Tese doutorado)

CASTELO BRANCO, M.; FRANÇA, F.H. Traça-das-crucíferas, Plutella xylostella (Lepidoptera: Yponomeutidae). In: VILELA, E.F.; ZUCCHI, R.A.; CANTOR, F., ed. Histórico das pragas introduzidas no Brasil. Ribeirão Preto: HOLOS, 2001. p.86-89.

CASTELO BRANCO, M.; FRANÇA, F.H.; MEDEIROS, M.A.; LEAL, J.G. Uso de inseticidas para o controle da traça-do-tomateiro e traçadas-crucíferas: um estudo de caso. Horticultura Brasileira, Brasília, v.19, n.1, p.60-63, 2001.

CASTELO BRANCO, M.; PONTES, L.A.; AMARAL, P.S.T.; MESQUITA, M.V. Inseticidas para o controle da traça-do-tomateiro e broca grande e impacto dos produtos sobre Trichogramma pretiosum. Horticultura Brasileira, Brasília, v.21, n.4, p.652-654, 2003.

CASTELO BRANCO, M.; GATEHOUSE, A.G. A survey of insecticide susceptibility in Plutella xylostella (L.) (Lepidoptera: Yponomeutidae) in the Federal District, Brazil. Neotropical Entomology, Londrina, v.30, p.327-332, 2001.

CASTELO BRANCO, M.; GATEHOUSE, A.G. Insecticide resistance in Plutella xylostella (Lepidoptera: Yponomeutidae) in the Federal District, Brazil. Anais da Sociedade Entomológica do Brasil, v.26, n.1, p.75-79, 1997.

CASTELO BRANCO, M.; MEDEIROS, M.A. Impacto de inseticidas sobre parasitóides de traça-das-crucíferas em repolho, no Distrito Federal. Pesquisa Agropecuária Brasileira, Brasília, v.36, n.1, p.7-13, 2001. 
CASTELO BRANCO, M.; VILLAS BÔAS, G.L.; FRANÇA, F.H. Nível de dano de traça das crucíferas em repolho. Horticultura Brasileira, Brasília, v.14, n.2, p.154-157, 1996.

FRANÇA, F.H.; MEDEIROS, M.A. Impacto de combinação de inseticidas sobre a produção de repolho e parasitóides associados com a traça-dascrucíferas. Horticultura Brasileira, Brasília, v.16, n.2, p.132-135, 1998.

GUAN-SOON, L. Overview of vegetable IPM in Asia. FAO Plant Protection Bulletin, v.38, p.7387. 1990.

HILL, T.A.; FOSTER, R.E. Effect of insecticides on the Diamondback Moth (Lepidoptera: Plutellidae) and its parasitoid Diadegma insulare (Hymenoptera: Ichneumonidae). Journal of Economic Entomology, v.93, n.3, p.763-768, 2000. IDRIS, A.B.; GRAFIUS, E. Field studies on the effect of pesticides on the diamondback moth (Lepidoptera: Plutellidae) and parasitism by Diadegma insulare (Hymenoptera: Ichneumonidae), 1993. Journal of Economic Entomology, v.86, n.4, p.1196-1202, 1993.
MILES, M.; DUTTON, R. Spinosad - a naturally derived insect control agent with potential for use in integrated pest management systems in greenhouses. The BCPC Conference: Pests and diseases. Volume 1. Proceedings of international conference held at the Brighton Hilton Metropole Hotel, Brighton, UK, 13-16 november, 2000 , p.339-344.

MONNERAT, R.G. Interrelations entre la teigne des cruciferes Plutella xylostella (L.) (Lep.: Yponomeutidae), son parasitoide; Diadegma $s p$ (Hym: Ichneumonidae) et la bacterie entomopathogene Bacillus thuringiensis Berliner Montpellier: École Nationale Superieure Agronomique de Montpellier, 1995. 162 p (Tese doutorado).

OLIVEIRA, A.T.; JUNQUEIRA, A.M.R.; FRANÇA, F.H. Impacto da irrigação por aspersão convencional na dinâmica populacional da traça-dascrucíferas em plantas de repolho. Horticultura Brasileira, Brasília, v.18, n.1, p.37-40. 2000.
RUBERSON, J.R.; TILLMAN, P.G. Effect of selected insecticides on natural enemies in cotton: laboratory studies. 1999. Proceedings Beltwide Cotton Conferences, Orlando, Florida, USA, 3-7, 1999: v.2., 1999, p.1210-1213.

SAMPSON, C. Producción de repolho en Belice: programa de investigación para el control de la palomilla dorso de diamante. Cerba, v.33, p.623628, 1992.

TAKASHINO, K.; NODA, T.; SAKAKIBARA, M. Effect of four insecticides on Diadegma semiclausum, a parasitoid of diamondback moth. $52^{\text {nd }}$ Annual Report on the Society of Plant Protection of North Japan, 2001, p.194-197. VILLAS BÔAS, G.L.; CASTELO BRANCO, M.; GUIMARÃES, A.L. Controle químico da traça das crucíferas em repolho no Distrito Federal. Horticultura Brasileira, Brasília, v.8, n.2, p.1011, 1990. 